\title{
石油分子表征与分子层次模型构建: 前沿及挑战
}

\author{
张霖宙, 赵锁奇, 史权, 徐春明* \\ 中国石油大学(北京)重质油国家重点实验室, 北京 102249 \\ *通讯作者, E-mail: xcm@cup.edu.cn
}

收稿日期: 2019-11-06; 接受日期: 2019-12-24; 网络版发表日期: 2020-2-14

国家重点研发计划(编号: 2018YFA0702400)和中国石油大学(北京)科研基金(编号: 2462018BJC003, 2462018QZDX04)资助项目

\begin{abstract}
摘要石油资源的绿色、高效及高值化利用需要从分子层次认识石油分子的组成及转化规律, 并构建分子层 次的精细化过程模型, 由此诞生了分子管理技术路线. 石油分子组成及转化过程的高度复杂性使得石油分子管理 技术开发面临巨大的挑战. 本文阐述了石油分子管理的沿革及内涵, 总结了石油分子组成表征及石油加工分子模 型的进展及所面临的挑战, 并对未来的发展方向进行了展望.
\end{abstract}

关键词石油加工, 分子管理, 分子表征, 分子层次模型, 过程优化

\section{1 引言}

对于现如今的石油炼化工业而言，过程模型已成 为不可或缺的内容. 在炼油化工过程中, 装置的设 计、开停工、生产排产及运行优化都需要模型进行辅 助定量计算. 长期以来, 炼油化工生产各环节中的设计 公司、工程公司、炼厂及科研院所都非常重视过程模 型的开发，大部分单位也都采购了流程模拟软件进行 过程模型开发. 随着对炼油化工过程优化要求的日益 提高, 学术界和工业界越来越明确地意识到, 精确的 过程模型对于炼油化工工业的进一步发展将起重要的 支撑作用.

在过去的几十年间，炼油化工工业的过程模型主 要基于简化的虚拟组分或者集总的方法来处理复杂石 油体系的分离及转化问题(在此称之为组分模型). 由 于生物前驱体本身具有的结构多样性，再加上成油过
程经历的复杂地质反应，石油含有数量巨大的分子类 型, 石油加工过程涉及的复杂反应转化网络更进一步 加剧了其复杂性. 因此, 石油转化过程的分子层次认 识和模拟对于研究者而言是一项巨大的挑战. 另一方 面, 石油工业对于过程模型的需求十分强烈, 迫使 20 世纪的科学家在石油化学及计算机硬件仍显不足的情 况下，提出可用于过程计算的简捷计算方法. 虚拟组 分/集总的方法以易获得的物理及化学信息为基础，根 据物理性质(如沸点)或化学特性(如四组分)将石油划 分成为有限的组分, 利用蒸馏或者柱色谱分离的方法 可以得到定量的组分信息，实现石油组成的特征化， 将石油加工过程中分子的分离及转化简化为有限数量 组分的分离及转化, 可计算获得分离及反应过程中的 已定义组分在产品中的收率及部分产品性质. 基于组 分的计算模型已有超过 80 年的历史 ${ }^{[1]}$, 现今仍是石油 加工过程模型构建的主流方法，商用流程模拟软件大

引用格式: Zhang L, Zhao S, Shi Q, Xu C. Molecular characterization and modeling of petroleum refining process: frontiers and challenges. Sci Sin Chim, 2020, 50: 192-203, doi: 10.1360/SSC-2019-0146 
多基于组分模型架构. 随着石化产品质量要求越来越 高, 炼油化工过程模型需要更高的预测能力及精细化 程度, 在此情况下组分模型的弊端逐渐显现, 主要体 现在: 无法满足产品关键质量的计算需求, 模型没有 包含过程机理而导致外推时预测能力不强, 分离及反 应过程由于组分定义不同难以进行模型衔接. 为了克 服传统组分模型的缺点, 学界在原有基础上进行了更 进一步的细化, 包括连续集总热力学模型及更细致的 组分划分，这些尝试部分解决了原有计算精度的问题， 但是没有从根本上解决组分模型的弊端.

分子层次模型由于其预测能力强、外推性好及可 以无缝进行模型连接等优点受到学界和工业界的重 视, 分子层次模型结合分子层次表征, 诞生了分子管理 技术方向. 分子是化工过程转化的基本单元, 基于分子 层次建立的模型反映了过程的化学本质, 具有良好的 预测性能及扩展性能. 过去由于受分析方法、计算机 硬件及分子层次模型化理论的限制, 石油加工过程的 分子层次模型构建难度较大, 相关的报道较少. 近 20 年来, 随着石油化学认识手段和计算机辅助复杂分子 体系模型构建方法的进步, 国际上涌现出一批从事石 油分子表征及分子层次模型化研究的课题组, 相关的 工业应用也逐步展开. 尽管分子层次模型复杂度高, 模型构建成本相比传统组分模型要大很多, 但是工业 初步应用结果表明其能够进一步提升计算的精确度和 精细度, 从而在包括原料选择、工艺设计、条件优化 及产品调合等方面取得更好的效果, 获取更高的收益. 在分子层次模型开发的过程中, 分子组成表征及分子 模型是相辅相成的, 分子组成表征为分子模型提供理 论基础及模型输入. 在新型复杂分子体系模拟理论的 基础上, 分子模型通过将分子表征的认识转化为定量 的模型, 提升炼油化工工业的整体优化水平. 本文简 述了石油分子层次表征及分子层次模型构建理论的进 展, 并指出了这些技术进一步发展需要应对的挑战.

\section{2 分子管理技术的发展历程及内涵}

石油分子表征是利用先进的仪器分析手段, 开发 适用于石油原料及产品的分子组成分析方法, 认识不 同来源石油原料及产品的组成结构特征分布, 揭示石 油加工过程中分子的分离及转化规律. 石油分子层次 模型化是在石油分子表征的基础上, 开发适用于石油
分子体系的模型化方法，实现石油特征分子重构，利 用计算机辅助方法开发石油加工过程的分子层次分离 及转化模型, 对石油加工环节进行模型调优, 从而实现 石油分子的最大化利用. 石油分子表征和石油分子层 次模型化技术是相互制约又相互促进的关系. 目前通 过适合的石油分子组成分析技术, 已经可以从较重的 馏分中鉴定出上万个分子, 在石油化学方面取得了较 大的认识, 而如此庞大的分子组成信息需要结合相适 应的分子层次模型进行信息处理及应用, 否则工业界 无法直接使用这些表征结果. 另一方面, 随着分子层 次模型精细化程度的提高, 需要分子表征提供更丰富 的定性及定量信息, 以指导模型构建、训练及输入, 反应转化模型的开发也需要基于可信的分子群转化 规律.

石油分子管理的理念最早见于ExxonMobil的技术 报告. ExxonMobil的科学家在石油分子表征及分子层 次模型构建理论两个领域都做出了很多开创性工作, 并且在商业上实现了较大成功，尽管ExxonMobil公司 较少与学界分享分子管理的技术细节, 但是其财报披 露的由分子管理带来的巨额利润极大地鼓舞了石油化 工学界及石油公司对于相关理论研发的热情. 美国高 磁国家实验室的Allan G. Marshall院士发明了傅里叶 变换离子回旋共振质谱(FT-ICR MS), 目前已广泛地应 用于重油复杂分子体系的组成解析, 另外其提出了石 油组学的概念(petroleomics), 指出基于分子层次信息 预测石油的物理性质及其转化性能的研究方向 ${ }^{[2,3]}$. 国 内何鸣元院士较早提出了分子炼油的概念, 倡导从分 子层次进行炼油化工过程的优化, 在炼油化工工业界 产生了深远的影响. 中国石油大学的重质油国家重点 实验室组建了石油分子工程中心, 围绕石油分子表征 及石油分子管理核心技术开展系统性研究, 提出了石 油分子组合与加工的概念, 着力打造一系列自有产权 技术并应用于石油加工过程优化.

炼油化工过程的分子层次模型构建最早可以追溯 到20世纪60年代, 主要集中于轻质油品的加工过程. 对 于石脑油加工过程, 由于其组成相对简单, 采用常规气 相色谱即可实现定性和定量分析. 由于反应及分离涉 及组分数较少, 早期分子层次模型构建的工作主要着 力于石脑油加工模型的开发, 人工处理分子数据库及 反应网络. 这些早期工作中较具有代表性的是SPYRO 模型及Mobil公司的KINPTR模型. SPYRO模型是面向 
乙烯裂解工艺开发的分子层次模型 ${ }^{[4,5]}$, 基于自由基层 面和分子路径层面编写的反应动力学模型, 实现了不 同原料、不同裂解炉型及不同工艺条件下产品分子收 率的准确计算. SPYRO模型在发表十几年后实现了商 业化，在其开发完成超过 50 年后的今天仍是乙烯裂解 工艺优化最常用的软件之一. KINPTR模型是Mobil公 司针对铂重整工艺开发的分子层次模型 ${ }^{[6]}$ ，历时约 5 年 时间完成, 采用了超过 300 个小试试验数据点进行了模 型训练, 由于该模型体现了过程的化学机理, 开发完成 后便迅速地被用于Mobil公司全球所有炼厂的重整工 艺优化及故障诊断，同时还用于实验室的工艺及催化 剂研发. 这两个模型的示例都非常明确地展示了分子 层次模型的强大预测能力、优化能力及生命力.

基于早期石脑油分子层次模型的商业成功，学界 和工业界开始进一步开发比石脑油更重馏分的分子层 次模型. 然而由于石油分子的复杂性, 石油分子表征和 分子层次模型开发很快便遇到了障碍. 以链烷烃为例, $\mathrm{C}_{10}$ 链烷烃的理论异构体数目是 75 , 常规的检测手段和 人工模型构建尚能处理如此规模的分子数量, 然而 $\mathrm{C}_{20}$ 链烷烃的理论异构体数目就攀升到超过 36 万, 并随着 碳数增加呈指数性上升. 石油中间馏分油及重油馏分 的分子复杂性都超出了早期石油分子表征和分子模型 构建能力的上限. 20 世纪70年代, Mobil公司发表了针 对较重石油加工工艺的集总模型 ${ }^{[7 ~ 9]}$, 集总模型开始成 为反应过程建模的主流，而同期开发的主流流程模拟 软件如Aspen Plus及HYSYS则选用了虚拟组分作为石 油蒸馏过程的主要模拟框架，炼油化工开始进入长期 由组分模型主导的时代. 分子层次模型再一次引起广 泛关注则是1990年前后由ExxonMobil公司的Steven B. Jaffe和Richard J. Quann及University of Delaware的 Michael T. Klein课题组在复杂分子体系建模理论方面 的引领性工作 ${ }^{[10 \sim 14]}$. 2000年以后，复杂体系分子表征 手段和分子层次理论的同步进展，在世界范围内再一 次掀起了炼油化工分子管理研究的热潮. 如今对于复 杂石油分子体系的分子表征和分子管理的研究依然处 于起步阶段. 尽管基础的理论已经有所突破, 但是对于 石油分子体系的组成结构及其行为还远远谈不上完全 认识, 分子层次的过程模型部分实现了工业应用, 但是 距离炼油化工过程完全的分子层次优化依然有距离, 相关的研究依然是工业界及学术界关注的重点及 难点.
如上所言, 石油分子体系的复杂性是石油分子管 理面临的主要挑战, 也是炼油化工过程模拟区别于精 细化工的重要特征, 石油分子管理近几十年来的研究 核心是如何解析及处理石油复杂分子体系及其转化行 为, 石油分子管理的关键问题是如何针对石油复杂分 子体系实现分子表征和分子层次模型化, 最终实现石 油复杂分子体系的认识及转化过程的精准优化, 其主 要研究内容如图1所示.

对于石油分子表征方面, 主要需要解决如下问题. (1) 石油分子体系的分子表征方法. 应用先进的分析仪 器, 针对石油加工过程的不同原料及产品, 开发针对性 的分子组成及分子结构的分析方法，实现不同石油馏 分的不同类型化合物的精确定性及定量解析. (2) 石 油分子及超分子体系的组成及结构认识. 针对石油分 子及其超分子聚集体，应用所开发的分子表征方法， 系统地认识石油中不同类型化合物的组成、结构及超 分子聚集体的特征，获取完整的分子群的分布及聚集 行为特征. (3) 石油加工过程中的分子转化规律. 针对 常见的炼油化工过程的分离及转化工艺, 研究石油分 子群的分离行为及转化规律, 揭示石油分子在分离过 程中的走向及在转化过程中的反应路径和反应活性, 为相关模型的开发提供重要理论基础.

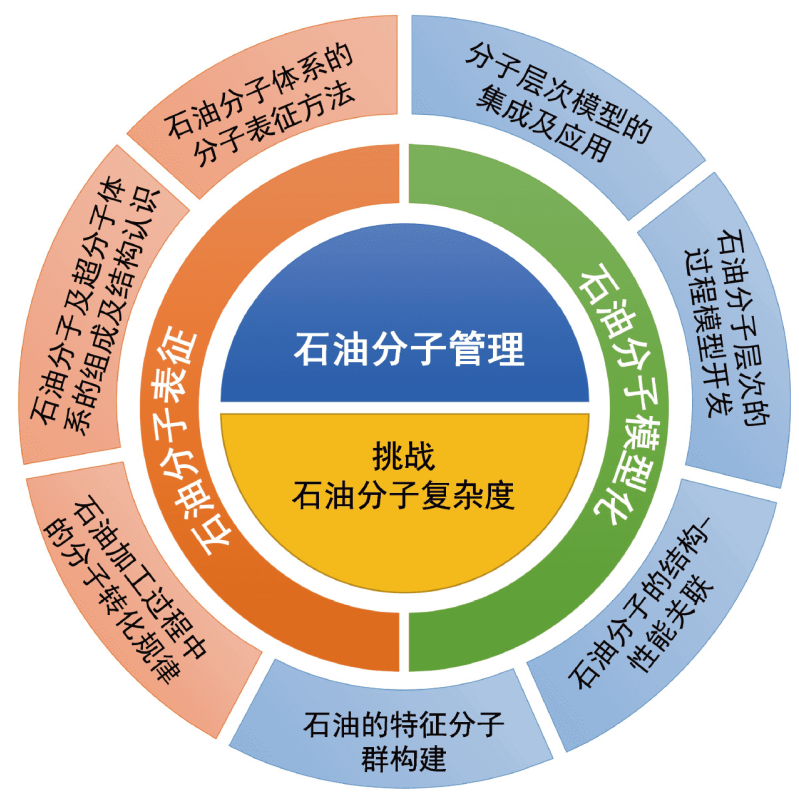

图 1 石油分子管理领域面临的主要挑战及主要研究内容 (网络版彩图)

Figure 1 The major challenges in the fields of petroleum molecular management (color online). 
对于石油分子模型化方面，主要需要解决如下问 题. (1) 石油的特征分子群构建. 综合石油分子表征所 得到的分子群分布特征，根据分离及转化过程描述的 精度需求，构建代表石油复杂分子群行为的特征分子， 并开发综合不同场景仪器分析结果的特征分子重构算 法, 实现特征分子群的求解. (2) 石油分子的结构-性能 关联. 针对石油分子体系, 基于机器学习方法开发石油 分子的结构-性能关联模型, 实现基于分子结构预测其 热力学及关键性质, 进而计算石油特征分子群的所有 关键性质，为过程模型的开发提供基础物性数据. (3) 石油分子层次的过程模型开发. 开发计算机辅助分子 层次模型构建方法，针对石油加工工艺过程特点，开 发复杂石油分子的分离及转化过程模型, 实现石油加 工过程的产品组成及性质的精准预测. (4) 分子层次 模型的集成及应用. 基于分子层次的认识及过程模型, 针对不同使用场景, 形成可行的工业化解决方案, 将模 型与炼厂的信息系统集成，并在工业场景进行分子层 次模型的应用, 最终实现石油加工过程的分子管理.

\section{3 石油分子组成表征进展}

原油是复杂有机化合物的混合物，其中含有烃 类、含氮、含氧、含硫及多杂原子化合物．石油按照 其沸点分布，可粗略划分为汽油、柴油、中间馏分油 及减压渣油等馏分，其中减压渣油中的沥青质组分由 于其在石油开采、运输及加工过程中引起的聚沉及生 焦等问题而引起广泛的关注. 石油分子的复杂程度随 着沸点的升高呈指数型上升. 汽油大约有几百个分子, 以烃类分子为主, 含有少量杂原子化合物, 而减压渣油 则约有数十万种分子, 富含多种稠环芳烃及杂原子化 合物. 长期以来，石油分子的复杂性对于分析化学学 科形成巨大的挑战，甚至有不少学者对认识石油复杂 分子体系的组成结构特征持悲观态度，然而随着石油 化学领域研究人员几十年来的持续努力，石油分子的 组成特征正被逐步揭示，不同石油馏分的复杂程度及 其对应的分子组成分析方法见图2，以下对几种代表 性的技术进行讨论.

汽油馏分由于其组成相对简单，采用常规的气相 色谱法即可实现大部分化合物的定性和定量分析. 针 对汽油中的烃类化合物，在 20 世纪 50 年代就发展了单 体烃分析方法，主要采用高分辨毛细管柱配合氢火焰

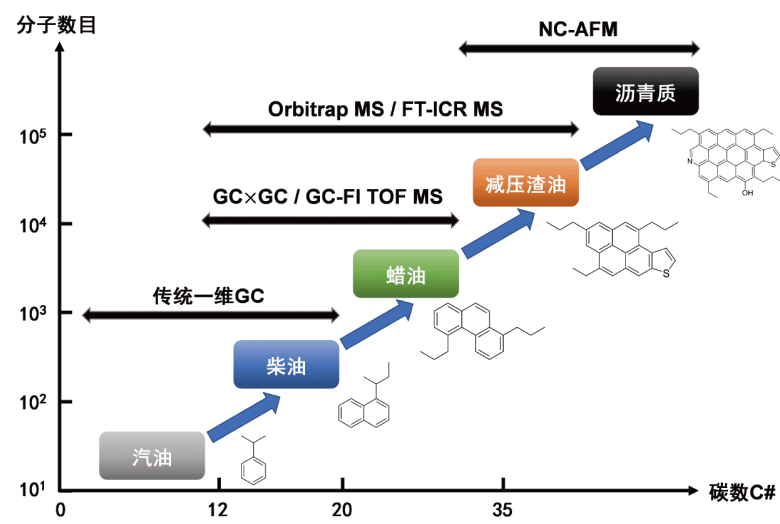

图 2 不同石油馏分的复杂程度及其对应的分子组成分析 方法(网络版彩图)

Figure 2 The complexity of different petroleum fractions and the corresponding analytical techniques (color online).

离子化检测器(FID)即可实现大部分化合物的定性及 定量分析, 在此称为常规一维气相色谱法. 而采用化学 发光检测器则可实现对汽油和柴油中含硫和含氮化合 物的选择性检测. 基于这些信息, 早期的研究者构建了 石脑油的分子转化模型. 然而随着馏分变重, 体系中分 子的数量超过了常规一维气相色谱法的分离极限(实 际上对于汽油中碳原子数超过 8 的分子已出现较为明 显的峰共逸出问题), 因此对于柴油及中间馏分已无法 实现单体烃的分离及定性定量分析，需要在传统一维 气相色谱的基础上增加新的维度.

针对柴油及中间馏分油的分子组成分析，学者们 开发了气相色谱和场电离源飞行时间质谱(GC FI$\mathrm{ToF}$ )技术. 通过气相色谱的预分离, 结合FI电离源的软 电离能力和 $\mathrm{ToF}$ 质谱的高分辨特性, 可以实现对绝大 部分烃类化合物的软电离及区分，从而获得族组成与 碳原子数的分布信息. 不同的化合物在FI电离源中具 有不同的电离效率, 因此GC FI-ToF还需要对所获得 的结果进行校正，从而获得准确的定量信息. 国外的 石油公司，如ExxonMobil及Chevron等，都基于 GC FIToF技术对柴油及中间馏分油进行了系统的研究，获 得了其组成分布特征 ${ }^{[15 ~ 18]}$. 国内的中国石油化工集团 有限公司的石油化工科学研究院也较早地进行了系统 性的方法开发 ${ }^{[19,20]}$. 在较长时间内, GC FI-ToF是柴油 及中间馏分油分子组成表征的常见方法. 然而, GC FIToF也存在一些缺点. 例如, 其直接采集的并非完全定 量信息, 需要对结果进行校正, 而采用的校正方法可能 
并不能准确获得定量信息. 此外, FI电离源的稳定性较 差, 降低了仪器操作的稳定性和重现性. GC FI-ToF在 分析仪器领域的份额正逐渐下降, Waters公司已经关 停了其GC FI-ToF生产线, 目前只有日本的JEOL公司 仍提供该仪器.

另外一种增加气相色谱维度的方法就是1990年后 出现的全二维气相色谱方法, 其利用调制器将两根独 立的色谱柱进行串联, 从而实现了化合物在两根色谱 柱上的二维分离, 极大地增加了气相色谱的分辨率、 灵敏度和峰容量, 在连接ToF质谱、FID检测器或选择 性的化学发光检测器后, 可对柴油及中间馏分油进行 准确定性及定量分析, 成为针对该体系进行定性定量 分析最适用的方法 ${ }^{[21 ~ 23]}$. 近30年来, 研究者基于全二 维气相色谱对石油馏分开发了检测方法, 成功地应用 于各类化合物的检测, 显示出良好的应用前景. 全二 维气相色谱的缺点是对于较重的中间馏分油, 其分离 能力可能依然无法满足要求, 存在一定的共逸出问题. 此外, 全二维气相色谱尚未形成广泛的可接受的标准 化方法, 其与分子层次模型的连接依然是值得研究的 课题.

基于气相色谱的方法仅能检测可汽化的石油馏 分, 无法检测减压渣油馏分, 对于减压渣油的分子组 成, 尤其是针对非烃化合物的组成分布特征, 长期以 来认识较少. 2000 年以后, ExxonMobil的Kuangnan Qian和美国高磁国家实验室的Allan G. Marshall和 Ryan P. Rodgers合作, 利用电喷雾电离源(ESI) FT-ICR MS成功实现了重油中含氮化合物的详细分子组成解 析, 从而开启了重油分子组成分析的新时代 ${ }^{[24,25]}$. 搭配 了超强磁场的FT-ICR MS 具有超高的分辨率和质量准 确度, 通过分子量可以精确地计算出其所对应谱峰的 分子式, 从而实现重油复杂分子组成的解析. 搭配各 种软电离技术, FT-ICR MS可以直接实现对重油中的 含氮、含氧及芳香类化合物的分子组成分布特征的分

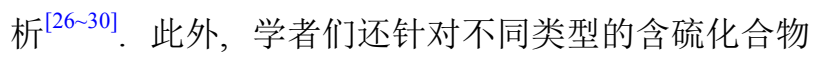
和含氮化合物等开发了化学衍生化方法, 实现对不同 类型化合物的精确识别 ${ }^{[31,32]}$. 学者们还应用了碰撞诱 导解离等技术分析了重油中的芳香环结构分布特征. 除了FT-ICR MS, 近期出现的静电场轨道阱质谱(Orbitrap MS)也具有类似的超高分辨率, 而且价格更为低 廉, 维护更为方便. 超高分辨质谱已经成为研究重油 分子组成的不可替代手段, 相关的文章也大量发表,
揭示了不同重油及其馏分的分离及转化特征, 极大地 丰富了重油化学的认识. 超高分辨率质谱目前的主要 瓶颈在于电离源技术, 主流的软电离技术只能电离一 部分重油分子, 而不同分子的电离选择性不同, 无法 获得定量的信息. 另外, 所得到的分子组成以分子式 表示, 无法区分同分异构体. 超高分辨质谱得到的定 性信息通常用以指导分子管理模型的分子选择及转化 路线指定, 但无法直接用于模型输入, 如何获取定量分 子组成信息是超高分辨质谱技术后续需解决的关键问 题之一.

对于重油化学研究而言, 分子结构的研究是更为 棘手的问题. 2012年, 来自IBM的Leo Gross课题组基 于超高分辨原子力显微镜的方法实现对芳香性分子的 结构解析, 成功地实现了分子成像 ${ }^{[33]}$. 随后各大石油 公司与Gross课题组合作进行重油(尤其是沥青质分子) 的分子结构研究 ${ }^{[3,35]}$. ExxonMobil公司近期也搭建了 自有的超高分辨原子力显微镜平台, 研究了模型化合 物及石油中高芳香性化合物的结构特征 ${ }^{[36-38]}$. 超高分 辨原子力显微镜直接得出了分子图像, 为沥青质结构 的认识提供了最为直观的证据, 引起了广泛关注, 所 得到的分子图像也为分子管理模型中分子结构的确定 提供了直接参考. 从分子管理角度来看, 该技术实验实 现难度较大, 仅能得到个别分子的结构, 无法提供分子 群的全貌, 也无法获得定量信息, 仅能作为定性的模型 信息输入.

图3展示了不同分子组成表征方法所得到的信息. 综上所述，石油分子组成表征沿着从轻馏分到重馏 分、从组成到结构方向不断进步，尽管石油分子具有 超高的复杂程度, 借助日益进步的分析技术及针对石 油分子体系开发的选择性分析方法, 石油分子的组成 结构分布特征正逐步被揭示, 从而可以更进一步获得 石油加工过程中分子的转化规律 ${ }^{[35,39 \sim 41]}$. 可以预见在 未来的进一步研究过程中, 将有望获得石油分子群完 整的定性和定量信息，从而为分子管理技术提供更为 直接且切实可靠的模型输入.

\section{4 石油加工分子层次模型化进展}

炼油化工分子管理技术应用的核心是分子层次模 型的开发, 石油分子表征技术的进步及所获得的石油 化学的认识为分子层次模型开发提供了重要的理论基 

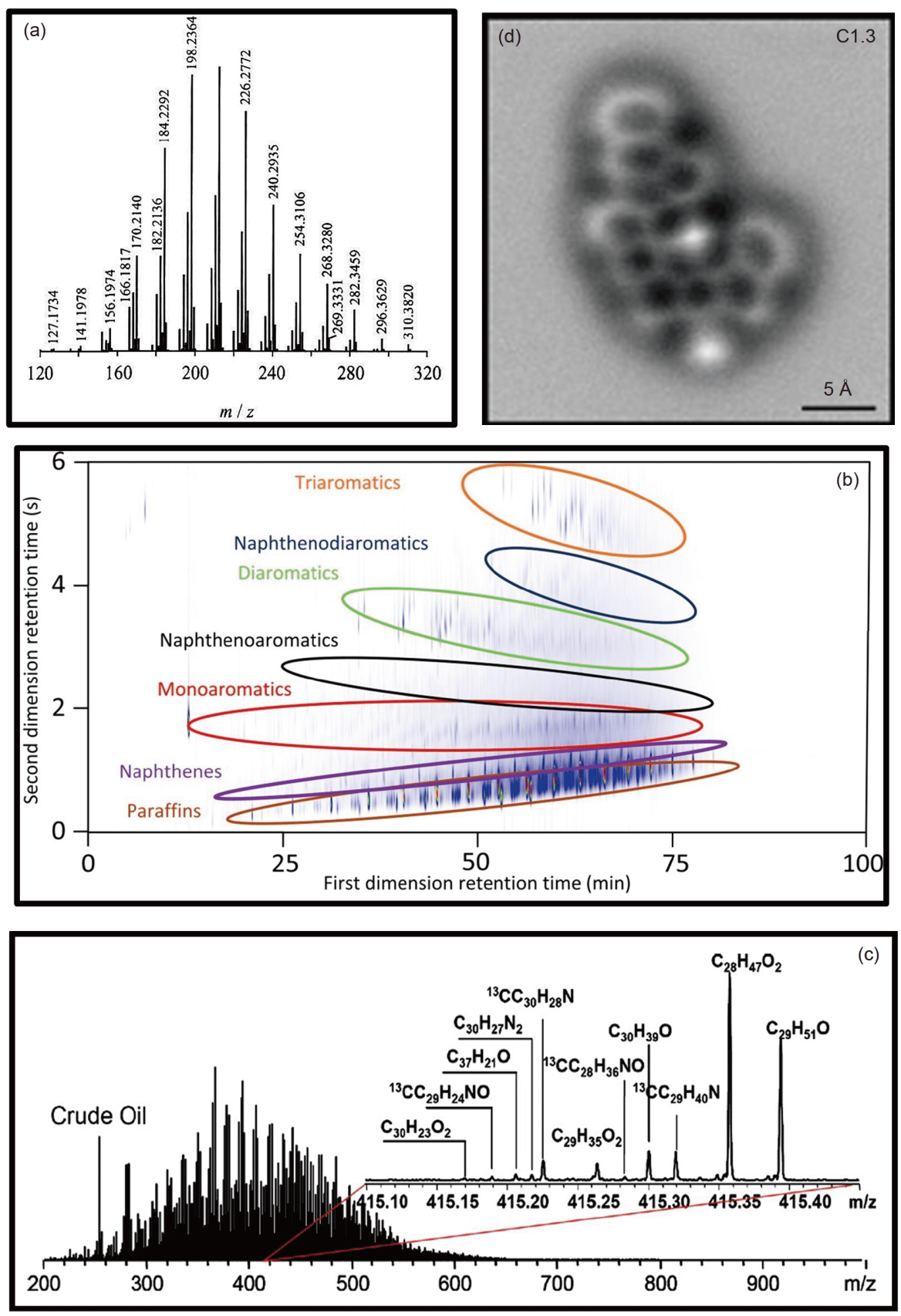

图 3 不同分子表征手段所得到的信息. (a) GC FI-ToF; (b) 全二维气相色谱; (c) FT-ICR MS; (d) 高分辨AFM图像 ${ }^{[35,39-41]}$ (网络 版彩图)

Figure 3 The information derived from different analytical measurements. (a) GC FI-ToF; (b) comprehensive two-dimensional GC; (c) FT-ICR MS; (d) high-resolution AFM image [35,39-41] (color online).

础，应用这些信息构建出准确、可靠及实用的分子层 次过程模型则是学界需要解决的另一重要问题.

石油分子转化过程按其机理可分为催化转化过程 和热转化两大类，其中主要涉及正碳离子机理和自由
基机理. 由于涉及大量的中间态及过渡态分子，化合物 的反应转化网络十分复杂, 此外还涉及分子在相间的分 配、传递、扩散及表面反应等多个步骤, 石油分子本身 便具有的超高复杂度, 若从机理层面来计算石油单一加 
工过程的转化路径, 其涉及的基本步骤数目将是天文数 字. 因而, 对于石油加工过程的模拟, 模型简化是十分必 要的. 而分子层次模型构建的关键环节之一是如何对分 子转化过程进行简化, 利用特征分子及其特征行为来代 表石油复杂分子群的复杂转化行为.

目前已有的石油加工过程的模型构建根据简化程 度的不同, 可分为多个模型构建尺度. 传统的方法是基 于平均分子和组分层面尺度, 其计算复杂度较低, 是目 前商用流程模拟软件的主流方法，但是其模型的精细 程度和描述能力较为不足. 目前许多研究者将精力投 入了分子层次模型的开发. 值得指出的是，分子层次 模型还可以按照其模型精细程度进行再一次细分. 通 常而言，模型的精细化程度升高则模型所涉及的分子 转化细节将增加，而同时模型的复杂程度、计算量和 构建难度也会随之增加. 按照涉及的模型的细化程度, 可分为: 预定义分子组分模型、基团层次模型、原子 连接模型和机理层次模型(自由基及正碳离子). 依照 对过程模型要求的精度及所开发的软件框架的计算能 力不同, 不同研究组在不同研究尺度进行了模型开发, 而不同尺度的模型适用的石油馏分的复杂度也不相 同, 图4展示了不同层次的石油加工模型及对应的分子 层次模型构建框架. 以下将对主流的模型尺度和模型
开发框架进行阐述, 主要围绕反应转化过程展开讨论.

早期的石油加工分子模型主要围绕石脑油过程展 开, 由于体系相对简单, 通常通过人工处理分子信息及 反应信息, 并手工推导反应网络, 编写反应过程的反应 动力学模型. 这些早期模型通常基于机理层面或者分 子路径层面, 通过对实验数据进行拟合, 可以求解反 应的动力学参数, 进而获得实用性及外推性都较强的 分子转化模型. 而随着馏分的变重, 所涉及的分子数 量呈指数上升, 无法人工处理复杂的反应网络, 对复 杂分子反应体系的建模方法提出了技术上挑战. 随后, 学界提出了计算机辅助模型构建方法, 其本质是利用 计算机来生成特征分子集, 利用结构-性质预测模型计 算分子性质，通过将反应机理转化为数字化的反应规 则，构建算法来推导特征分子所具有的特征反应，并 自动编写反应动力学模型, 以实现复杂分子体系反应 模型的构建. 目前, 主流的分子管理技术研发课题组 基本都是基于计算机辅助的方法，通过自主研发的分 子描述方法及框架进行模型构建.

目前商业化最成功的分子层次模型化方法是 1990 年左右由ExxonMobil的Steven B. Jaffe及Richard J. Quann提出的结构导向集总方法 $(\mathrm{SOL})^{[10 \sim 12]}$. SOL是一 种基于基团层面进行石油分子层次模型构建的方法,

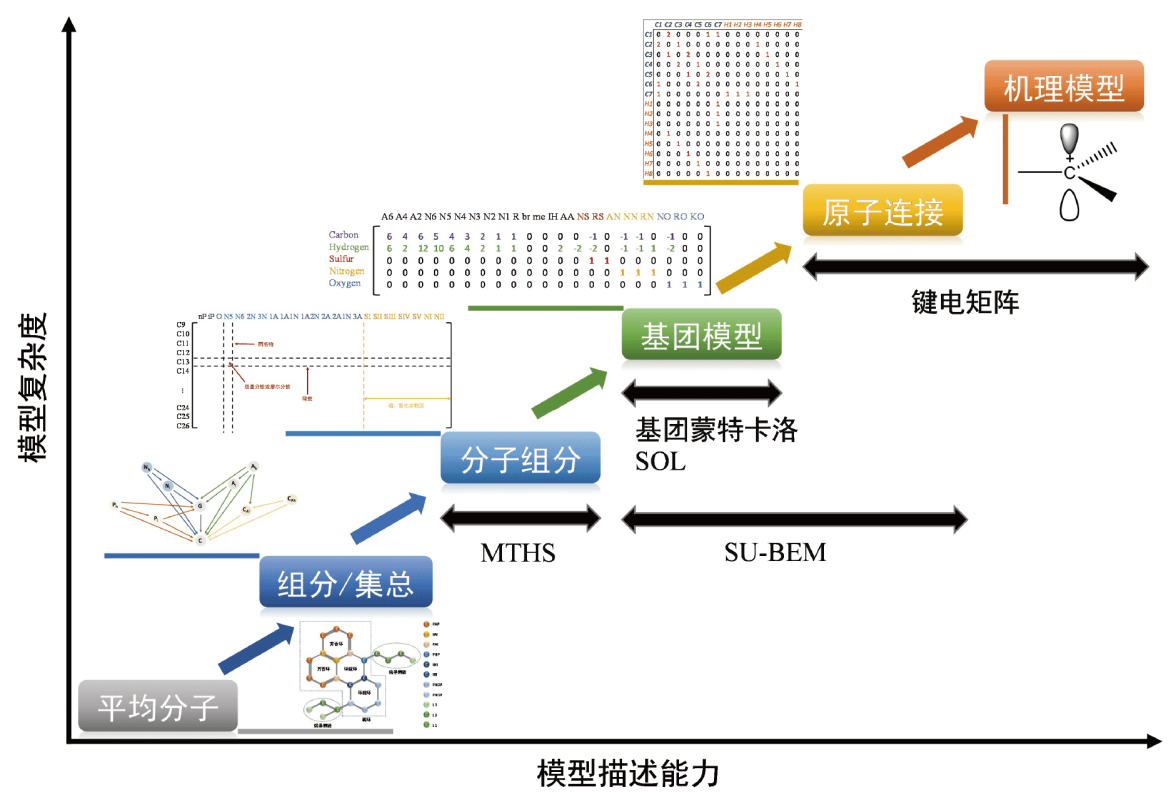

图 4 不同层次的石油加工模型及对应的分子层次模型构建框架(网络版彩图)

Figure 4 The different level petroleum refining model and the corresponding model building framework (color online). 
通过总结GC FI-ToF得到的详细组成及转化规律信息, 预定义一系列结构单元，利用不同单元的组合构筑不 同的石油分子，而石油分子的反应则由基团的变化表 示. SOL框架在与分子表征连接、模型的复杂程度及 化学直观性三方面取得了平衡点, 分子及反应定义方 便，数学化表述简洁，同时又能与馏分油的分子表征 结果直接结合. ExxonMobil的团队基于SOL开发了自 动化的分子组成微调算法、独有的分子性质计算方 法、反应网络生成算法及反应动力学代码生成算法, 打通了计算机辅助分子层次模型构建的全流程，构建 了不同石油加工过程的分子层次模型, 通过GC FID或 GC FI-ToF直接获得模型输入, 可准确预测产品的分子 组成和关键性质, 形成了自有的过程模型软件, 在公司 内部进行了大量应用, 取得了商业上的成功, 因而广受 关注. 国内的华东理工大学也采用该框架完成了自有 的模型化方法开发，并完成了一系列石油馏分的分子 组成模型及加工过程模型开发 ${ }^{[42-45]}$.

与SOL同时期, 来自University of Delaware的Klein 课题组 ${ }^{[13]}$ 也基于基团层面开发了基于自定义的基团 结合蒙特卡洛模拟，实现了石油馏分的分子组成模型 构建及反应过程模型构建. Klein课题组在这一时期的 另一主要贡献是确立了基于宏观性质重构分子组成模 型的基本方法, 包括基于 Gamma分布的分子抽样和通 过全局优化算法进行与试验性质对比的多目标优化 等, 这些方法至今仍被广泛采用. 自定义基团结合蒙特 卡洛法也被其他课题组采用，如加拿大国家能源中心 $(\mathrm{NRC})^{[46]}$ 和法国石油研究所(institute of petrole France, IFP). IFP还进一步提出了最大熵的二次调节算法，进 一步提高了模型精度 ${ }^{[47}$. 0$]$.

基团层面的模型虽然简洁且易于处理，但是无法 描述石油分子的精细结构，如甲基的位置等. Klein课 题组 ${ }^{[51 \sim 54]}$ 在提出自定义基团结合蒙特卡洛法后，进一 步升级了模拟框架，从基团层面进一步细化到原子拓 扑层面. 通过定义键电矩阵(BE matrix), 可以实现分 子结构及电荷位置的详细描述，在此基础上发展出一 系列键电矩阵的生成及操作算法, 通过键电矩阵对原 子所处环境进行判断，实现自动化反应网络构建，同 期还提出了线性自由能方法(LFER)及结构反应性关 联方法(QSRC)对反应过程的参数进行降维, 成为当前 复杂反应网络求解的常用策略 ${ }^{[54]}$. 在键电矩阵的基础 上, Klein课题组 ${ }^{[5]}$ 开发了KMT软件包，并对多种石油
加工过程进行模拟。自2000年以后，Klein课题组已不 再采用自定义基团结合蒙特卡洛的方法，而是全面采 用键电矩阵方法进行模型构建. 基于键电矩阵的强拓 展性, Klein课题组还进行了煤转化、生物质转化等过 程模型的开发. 键电矩阵作为原子及机理层面的模拟 框架, 具有较强的描述能力, 但是其处理难度大, 对于 模型开发者的化学及数学要求较高. 目前仅有美国西 北大学的Linda Broadbelt及麻省理工大学的Willam Green课题组能使用类似的方法进行建模.

石油分子层次模型的另一个思路是将原有的组分 模型进行进一步拓展, 引入更多的分子信息, 形成预定 义分子组分来进行模型的构建，该方法通常将石油组 成表示成矩阵的形式，矩阵中的每个单元代表一个预 定义分子组分，不同的石油馏分仅表现为矩阵值的不 同，而反应则表示为矩阵中单元的转换. 这种方法与 轻馏分的分子组成检测数据相匹配，易于计算机实现. 曼彻斯特大学的过程集成中心基于这种思想提出了分 子类型同系物矩阵(MTHS)的框架 ${ }^{[6,57]}$, 发展了一系列 以此为基础的分子集重构及反应模型构建方法. MTHS方法一开始仅用于汽柴油等较轻馏分的模型构 建, 随后被拓展到较重的石油馏分, 同时也进行了更进 一步的简化 ${ }^{[58]}$. IFP基于类似的思想，也提出了虚拟化 合物矩阵的方法, 进行了馏分油的构建 ${ }^{[59]}$. 预定义分 子组分的方法相比于传统的组分方法(虚拟组分和集 总)，集成了更多的分子信息，而采用了类似的建模方 法进行建模, 这些工作也表明, 高度简化的分子模型和 高度细化的组分模型间并没有清晰的分割线，集成更 多的分子信息也是传统组分模型升级的一个可行 方法.

机理层次模型最接近反应转化过程的本质, 因而 其模型的外推性最好, 然而模型的复杂程度最高. 机 理模型的代表之一是用于乙烯裂解过程的SPYRO模 型，而SPYRO模型到现在依然被广泛采用，证明了准 确机理模型的优越性 ${ }^{[4]}$. 同时, 机理模型还可以通过量 子化学计算的方法获得每个步骤的过渡态和活化能, 理论上可以完全通过第一性原理计算获得每个步骤的 反应速率 ${ }^{[60]}$. Froment课题组 ${ }^{[61 \sim 63]}$ 基于机理模型提出了 单事件方法(Single-Events)来计算过程速率，并且进行 了石油加工过程的模型开发, 取得了良好的效果. 然而 机理模型导致的反应数目爆发性增加的问题十分显 著, 对于复杂石油分子体系而言, 反应网络的简化及后 
处理是机理模型需要解决的问题, 目前对于较重石油 馏分的加工过程较少采用机理模型进行构建, 已有报 道大多是方法学上的探索性工作.

不同层级的分子层次模型具有各自的优缺点，总 体而言，包含细节越少的分子模型越易于构建和处理, 但是模型的描述能力越差, 反之亦然. 例如, 基于 SOL 的模型易于理解和计算，但是由于不包含原子拓扑信 息, 模型的拓展性不强; 而另一方面, 基于 $\mathrm{BE}$ matrix的 方法虽然能够完整描述键和原子的连接关系，但是处 理起来却十分困难. 为此, 本课题组 ${ }^{[64]}$ 提出了混合式 的石油加工的分子模型化方法，即混合式的结构单元 和键电矩阵方法(SU-BEM), 其基本思想是通过构建不 同模型之间的转换方法, 在单一模型内同时集成“分子 组分-结构单元-原子拓扑-键电矩阵”信息(图5). 为此, 本课题组开发了完整的软件平台，实现基于任一层次 模型定义, 自动进行模型转化, 从而实现灵活的模型 构建. 基于此方法，我们构建了从汽油到重油的分子 组成模型、蒸馏过程模型、溶剂萃取模型、分子转化
模型及产品调合模型，打通了石油分子管理技术的全 链条 ${ }^{[65]}$.

总的来说, 目前石油加工分子模型化主要是以计 算机辅助模型构建为主流技术，根据模型描述的精细 程度不同，模拟方法和框架呈现“百花齐放”的状态. 不同的课题组都具有自有的软件框架并开发了不同的 过程模型, 而下一步研究工作的主要着力点是如何进 行工业应用, 将实验室原型用于实际生产过程中.

\section{5 展望}

石油分子管理严格而言并非全新的石油加工过程 模拟和优化技术，是随着石油分子组成表征技术和复 杂分子层次模型化理论的进步，由传统的虚拟组分/集 总模型发展而至的必然的技术路线，其本质是在认识 石油分子组成及转化规律的基础上，在分子层次模拟 石油特征分子群的组成及转化行为. 石油分子管理技 术是炼油化工行业朝着高效、精细和绿色化的过程设

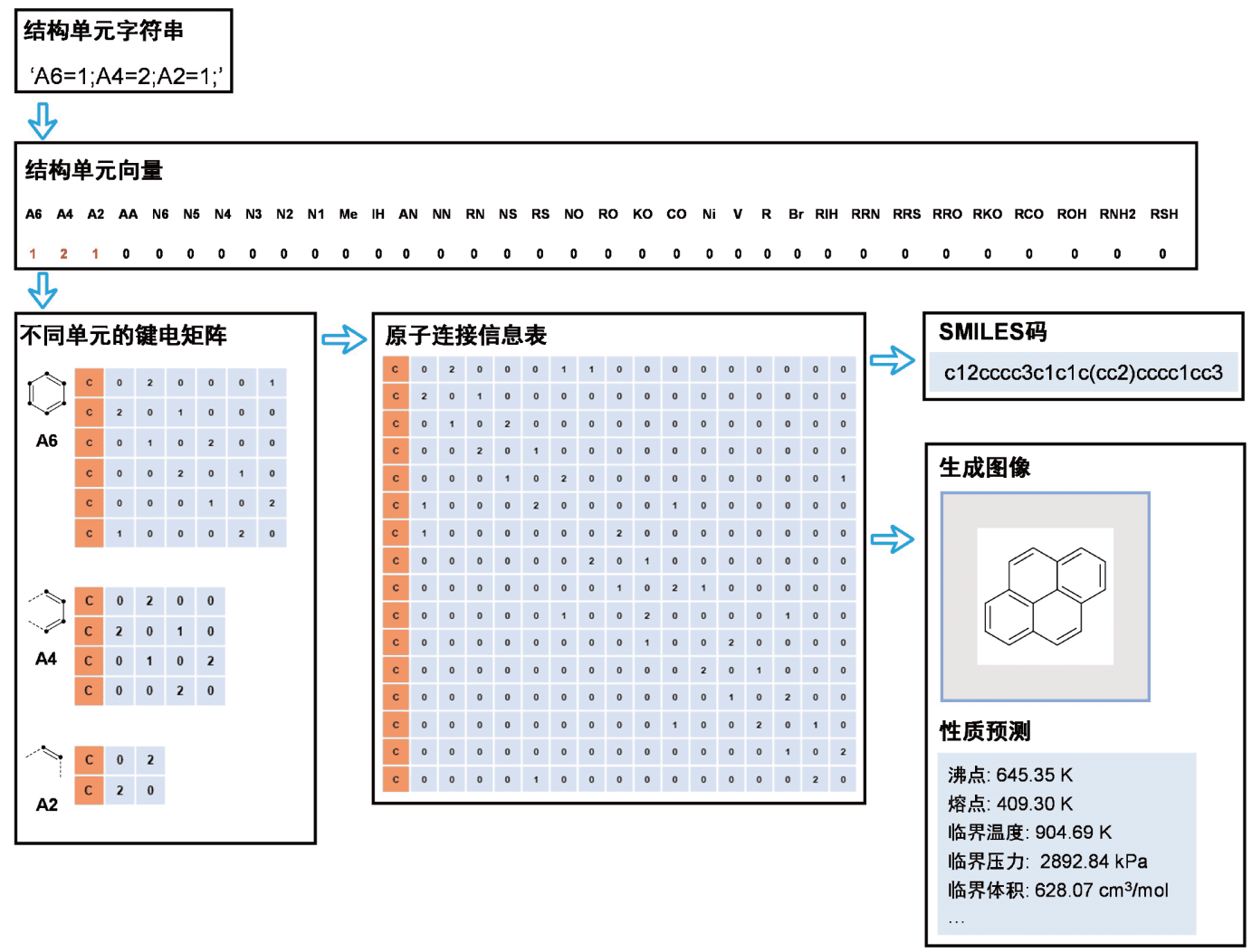

图 5 中国石油大学开发的混合式结构单元和键电矩阵(SU-BEM)框架(网络版彩图)

Figure 5 Combinational structural unit and bond-electron matrix (SU-BEM) frame developed by our research group (color online). 
计、调控及优化发展的必经之路. 我们认为, 在未来的 发展历程中，石油分子表征及分子模型化将沿着以下 方向继续发展.

(1) 分子组成表征. 发展适用性更强的分子组成表 征方法, 准确解析石油分子的组成、官能团及结构特 征，获得石油分子(尤其是重油分子)准确的定性及定 量结果; 更全面地认识石油分子的组成及结构分布, 完整揭示石油分子转化过程的主要分子行为, 形成常
见石油原料及产品的分子组成数据库；发展更为快速 的石油分子表征方法, 为工业在线应用提供检测技术;

(2) 分子模型化方法. 发展更为灵活及现代化的分 子层次模型构建框架, 构建更为准确及快速的组成模 型及过程模型，耦合更多的机理信息，实现模型训练 需求实验数据的减少, 进一步拓宽模型的应用范围, 与计算流体力学及智能化炼厂信息平台集成，提供更 精细的优化能力.

\section{参考文献}

1 Katz DL, Brown GG. Ind Eng Chem, 1933, 25: 1373-1384

2 Marshall AG, Rodgers RP. Acc Chem Res, 2003, 37: 53-59

3 Marshall AG, Rodgers RP. Proc Natl Acad Sci USA, 2008, 105: 18090-18095

4 Dente M, Ranzi E, Goossens AG. Comput Chem Eng, 1979, 3: 61-75

5 van Goethem MWM, Kleinendorst FI, van Leeuwen C, van Velzen N. Comput Chem Eng, 2001, 25: 905-911

6 Ramage MP, Graziani KR, Schipper PH, Krambeck FJ, Choi BC. Advances in Chemical Engineering. Amsterdam: Elsevier, 1987. 193-266

7 Weekman VW, Nace DM. AIChE J, 1970, 16: 397-404

8 Nace DM, Voltz SE, Weekman VW. Ind Eng Chem Proc Des Dev, 1971, 10: 530-538

9 Jacob SM, Gross B, Voltz SE, Weekman VW. AIChE J, 1976, 22: 701-713

10 Quann RJ, Jaffe SB. Ind Eng Chem Res, 1992, 31: 2483-2497

11 Quann RJ, Jaffe SB. Chem Eng Sci, 1996, 51: 1615-1635

12 Jaffe SB, Freund H, Olmstead WN. Ind Eng Chem Res, 2005, 44: 9840-9852

13 Neurock M, Libanati C, Nigam A, Klein MT. Chem Eng Sci, 1990, 45: 2083-2088

Zhu XY, Liu ZL, Xu YQ, Liu YR, Tian SB. Acta Petrol Sin (Petrol Proc Sect), 2011, 27: 797-800 (in Chinese) [祝馨怡, 刘泽龙, 徐延勤, 刘颖 荣, 田松柏. 石油学报(石油加工), 2011, 27: 797-800] ing JJ, Liu YR, Liu ZL, Tian SB. Chin J Analy Chem, 2016, 44: 416-422 (in Chinese) [蒋婧婕, 刘颖荣, 刘泽龙, 田松柏. 分析化学, 2016, 44: 416-422]

21 Dutriez T, Courtiade M, Thiébaut D, Dulot H, Hennion MC. Fuel, 2010, 89: 2338-2345

Qian K, Robbins WK, Hughey CA, Cooper HJ, Rodgers RP, Marshall AG. Energy Fuels, 2001, 15: 1505-1511

Qian K, Rodgers RP, Hendrickson CL, Emmett MR, Marshall AG. Energy Fuels, 2001, 15: 492-498

Rodgers RP, Hendrickson CL, Emmett MR, Marshall AG, Greaney M, Qian K. Can J Chem, 2001, 79: 546-551

Schaub TM, Hendrickson CL, Quinn JP, Rodgers RP, Marshall AG. Anal Chem, 2005, 77: 1317-1324

Hsu CS, Hendrickson CL, Rodgers RP, McKenna AM, Marshall AG. J Mass Spectrom, 2011, 46: 337-343

Shi Q, Xu C, Zhao S, Chung KH, Zhang Y, Gao W. Energy Fuels, 2010, 24: 563-569

Shi Q, Hou D, Chung KH, Xu C, Zhao S, Zhang Y. Energy Fuels, 2010, 24: 2545-2553

Liu P, Xu C, Shi Q, Pan N, Zhang Y, Zhao S, Chung KH. Anal Chem, 2010, 82: 6601-6606 
Zhou X, Shi Q, Zhang Y, Zhao S, Zhang R, Chung KH, Xu C. Anal Chem, 2012, 84: 3192-3199

Gross L, Mohn F, Moll N, Liljeroth P, Meyer G. Science, 2009, 325: 1110-1114

Schuler B, Meyer G, Peña D, Mullins OC, Gross L. J Am Chem Soc, 2015, 137: 9870-9876

Schuler B, Fatayer S, Meyer G, Rogel E, Moir M, Zhang Y, Harper MR, Pomerantz AE, Bake KD, Witt M, Peña D, Kushnerick JD, Mullins OC, Ovalles C, van den Berg FGA, Gross L. Energy Fuels, 2017, 31: 6856-6861

Zhang Y. Energy Fuels, 2019, 33: 3816-3820

Zhang Y, Schuler B, Fatayer S, Gross L, Harper MR, Kushnerick JD. Ind Eng Chem Res, 2018, 57: 15935-15941

Zhang Y, Schulz F, Rytting BMK, Walters CC, Kaiser K, Metz JN, Harper MR, Merchant SS, Mennito AS, Qian K, Kushnerick JD, Kilpatrick PK, Gross L. Energy Fuels, 2019, 33: 6088-6097

Zhu XY, Liu ZL, Xu YQ, Liu YR, Tian SB. Acta Petrol Sin (Petrol Proc Sect), 2010, 26: 277-282 (in Chinese) [祝馨怡, 刘泽龙, 徐延勤, 刘颖 荣, 田松柏. 石油学报(石油加工), 2010, 26: 277-282]

Dijkmans T, Van Geem KM, Djokic MR, Marin GB. Ind Eng Chem Res, 2014, 53: 15436-15446

Shi Q, Zhao S, Xu Z, Chung KH, Zhang Y, Xu C. Energy Fuels, 2010, 24: 4005-4011

Ni TY, Liu JC, Shen BX, Sun H. Petrol Proc Petrochem, 2015, 46: 15-22 (in Chinese) [倪腾亚, 刘纪昌, 沈本贤, 孙辉. 石油炼制与化工, 2015, 46: $15-22]$

Zhu R, Shen BX, Liu JC. Petrol Proc Petrochem, 2013, 44: 37-42 (in Chinese) [祝然, 沈本贤, 刘纪昌. 石油炼制与化工, 2013, 44: 37-42] Shen BX, Tian LD, Liu JC. Acta Petrol Sin (Petrol Proc Sect), 2010, 26: 218-225 (in Chinese) [沈本贤, 田立达, 刘纪昌. 石油学报(石油加工), 2010, 26: 218-225]

Tian L, Shen B, Liu J. Energy Fuels, 2012, 26: 1715-1724

Alvarez-Majmutov A, Gieleciak R, Chen J. Fuel, 2019, 241: 744-752

de Oliveira LP, Vazquez AT, Verstraete JJ, Kolb M. Energy Fuels, 2013, 27: 3622-3641

de Oliveira LP, Verstraete JJ, Kolb M. Chem Eng J, 2012, 207-208: 94-102

Verstraete JJ, Schnongs P, Dulot H, Hudebine D. Chem Eng Sci, 2010, 65: 304-312

Hudebine D, Verstraete JJ. Oil Gas Sci Technol-Rev IFP Energies Nouvelles, 2011, 66: 437-460

Broadbelt LJ, Stark SM, Klein MT. Ind Eng Chem Res, 1995, 34: 2566-2573

Joshi PV, Freund H, Klein MT. Energy Fuels, 1999, 13: 877-880

Joshi PV, Kumar A, Mizan TI, Klein MT. Energy Fuels, 1999, 13: 1135-1144

Klein MT. Molecular Modeling in Heavy Hydrocarbon Conversions. Boca Raton: CRC/Taylor \& Francis, 2006

Wei W, Bennett CA, Tanaka R, Hou G, Klein MT Jr., Klein MT. Fuel Proc Tech, 2008, 89: 350-363

Gomez-Prado J, Zhang N, Theodoropoulos C. Energy, 2008, 33: 974-987

Peng B. Molecular modelling of petroleum processes. Dissertation for the Doctoral Degree. Manchester: University of Manchester, 1999

Liu L. Molecular characterisation and modelling for refining processes. Dissertation for the Doctoral Degree. Manchester: University of Manchester, 2015

Hudebine D, Verstraete J, Chapus T. Oil Gas Sci Technol-Rev IFP Energies Nouvelles, 2011, 66: 461-477

Sabbe MK, Van Geem KM, Reyniers MF, Marin GB. AIChE J, 2011, 57: 482-496

Froment G. Catal Today, 1999, 52: 153-163

Dewachtere NV, Santaella F, Froment GF. Chem Eng Sci, 1999, 54: 3653-3660

Feng W, Vynckier E, Froment GF. Ind Eng Chem Res, 1993, 32: 2997-3005

Feng S, Cui C, Li K, Zhang L, Shi Q, Zhao S, Xu C. Chem Eng Sci, 2019, 201: 145-156

Zhang L, Chen Z, Lyv W, Li K, Cui C, Shi Q, Zhao S, Xu C. Sci Sin Chim, 2018, 48: 411-426 (in Chinese) [张霖宙, 陈政宇, 吕文进, 李凯宇, 崔 晨, 史权, 赵锁奇, 徐春明. 中国科学:化学, 2018, 48: 411-426] 


\title{
Molecular characterization and modeling of petroleum refining process: frontiers and challenges
}

\author{
Linzhou Zhang, Suoqi Zhao, Quan Shi, Chunming Xu* \\ State Key Laboratory of Heavy Oil Processing, China University of Petroleum, Beijing 102249, China \\ *Corresponding author (email: xcm@cup.edu.cn)
}

\begin{abstract}
The green, high efficient and high value-added utilization of petroleum resource requires the molecular management of refining process. As the basis of precisely controlling and optimization of refinery, the molecular-level characterization and modeling of the petroleum and the related conversion process are widely concerned. The complexity of petroleum composition and conversion pathway form significant challenge for the development of molecular management technologies. In the presented paper, we summarized the progress in the fields of petroleum molecular-level characterization and modeling, and the researches that needed in the future were also prospected.
\end{abstract}

Keywords: petroleum refining, molecular management, molecular characterization, molecular modeling, process optimization

doi: $10.1360 /$ SSC-2019-0146 